COMMUNICATIONS IN

ANALYSIS AND GEOMETRY

Volume 14, Number 4, 765-793, 2006

\title{
Geometry of moduli spaces of Higgs bundles
}

\author{
Indranil Biswas and Georg Schumacher
}

\begin{abstract}
We construct a Petersson-Weil-type Kähler form on the moduli spaces of Higgs bundles over a compact Kähler manifold. A fiber integral formula for this form is proved, from which it follows that the Petersson-Weil form is the curvature of a certain determinant line bundle, equipped with a Quillen metric, on the moduli space of Higgs bundles over a projective manifold. The curvature of the Petersson-Weil Kähler form is computed. We also show that, under certain assumptions, a moduli space of Higgs bundles supports of natural hyper-Kähler structure.
\end{abstract}

\section{Introduction}

A Higgs bundle over a compact Kähler manifold $X$ is a pair of the form $(E, \varphi)$, where $E$ is a holomorphic vector bundle over $X$ and $\varphi$ a holomorphic section of $\operatorname{End}(E) \otimes \Omega_{X}^{1}$ satisfying the integrability condition $\varphi \wedge \varphi=0$. Higgs bundles over a compact Riemann surface were introduced by Hitchin in [1], where he constructed their moduli and investigated the global, as well as the local, structures of the moduli space. One of the main results of [1] was that a stable Higgs bundle admits a unique Hermitian-Yang-Mills connection. Simpson, initiating the study of Higgs bundles over compact Kähler manifolds of arbitrary dimension, proved that a stable Higgs bundle admits a unique Hermitian-Yang-Mills connection [2]. He also constructed the moduli space of Higgs bundles over a complex projective manifold [3].

The aim in this article is to study the local geometry of a moduli space of Higgs bundles from the point of view of the generalized Petersson-Weil geometry, which has been carried out for the moduli spaces of stable vector bundles $[4,5]$. Here, a moduli space is by definition a reduced complex space. With additional effort, also non-reduced moduli spaces can be investigated.

For any Higgs bundle $(E, \varphi)$ over a compact Kähler manifold $X$, there is an associated complex of $\mathcal{O}_{X}$-modules

$$
\begin{aligned}
D^{\bullet}: D^{0} & :=\operatorname{End}(E) \longrightarrow D^{1}:=\operatorname{End}(E) \otimes \Omega_{X}^{1} \longrightarrow \cdots \longrightarrow \\
D^{i} & :=\operatorname{End}(E) \otimes \Omega_{X}^{i} \longrightarrow \cdots
\end{aligned}
$$


with the homomorphisms defined by $s \mapsto[s, \varphi]$. The global endomorphisms and the infinitesimal deformations of $(E, \varphi)$ are given by the hypercohomologies $\mathbb{H}^{0}\left(D^{\bullet}\right)$ and $\mathbb{H}^{1}\left(D^{\bullet}\right)$, respectively. Similarly, the obstructions for deformations of $(E, \varphi)$ are guided by $\mathbb{H}^{2}\left(D^{\bullet}\right)$. We consider the Dolbeault resolution of the above complex $D^{\bullet}$. So the hypercohomologies of $D^{\bullet}$ get identified, with the cohomologies associated to the resulting double complex.

Now, the existence of Hermitian-Yang-Mills connections on stable Higgs bundles allows us to introduce a natural inner product on the terms of the above mentioned double complex, and the general Hodge theory provides the space of hypercohomologies with a Hermitian structure. In other words, it is possible to identify harmonic representatives of the hypercohomologies, and the Hermitian structure on hypercohomologies is given by the Hermitian structure on the harmonic representatives. In particular, the space of infinitesimal deformations of a stable Higgs bundle $(E, \varphi)$ is equipped with a natural Hermitian structure. In this way, any moduli space of Higgs bundles over $X$ is provided with a natural Hermitian metric.

This Hermitian structure on a moduli space of Higgs bundles is actually a Kähler structure (Proposition 4.2); we call this Kähler form the generalized Petersson-Weil form. We compute the curvature tensor of this generalized Petersson-Weil form (Theorem 5.1). For a moduli space Higgs bundles over a compact Riemann surface, the holomorphic sectional curvature turns out to be non-negative (Corollary 5.3).

Furthermore, we prove a fiber integral formula for the generalized Petersson-Weil form (see Proposition 6.1 and Theorem 6.2). The fiber integral formula implies the Kähler property also for families parameterized by singular base spaces. Finally, the generalized Riemann-Roch theorem of Bismut, Gillet and Soulé provides a certain determinant line bundle equipped with a Quillen metric over the moduli space of Higgs bundles whose curvature form coincides with the generalized Petersson-Weil form on the moduli space (Theorem 6.3).

In Section 7, we construct a distinguished locally exact holomorphic 2 -form $\pi$ on the moduli space of Higgs bundles. In order to show nondegeneracy of $\pi$, we need an involution $\iota$, on the first hypercohomology, defined in terms of harmonic representatives. To construct $\iota$, we need two assumptions on $(E, \varphi)$ : (1) the rational characteristic classes of the projective bundle $\mathbb{P}(E)$ vanish and $(2) \operatorname{dim} \mathbb{H}^{2}\left(D^{\bullet}\right)=1$.

We note that, in general, $\operatorname{dim} \mathbb{H}^{2}\left(D^{\bullet}\right) \geq 1$. Hence, by semi-continuity, the condition $\operatorname{dim} \mathbb{H}^{2}\left(D^{\bullet}\right)=1$ defines a Zariski open subset of any moduli space of Higgs bundles. We also note that, in general, $\operatorname{dim} \mathbb{H}^{1}\left(D^{\bullet}\right)$ may be odd. 
As an application, we construct a hyper-Kähler structure on the moduli space using $\iota$, the generalized Petersson-Weil form and $\pi$ (Theorem 8.6).

\section{Basic definitions}

Let $X$ be a compact, connected Kähler manifold of dimension $n$ equipped with a Kähler form $\omega_{X}$. We will write $\omega_{X}=\sqrt{-1} g_{\alpha \bar{\beta}} d z^{\alpha} \wedge d z^{\bar{\beta}}$ with respect to local holomorphic coordinates $\left(z^{1}, \ldots, z^{n}\right)$, and we will always use the summation convention.

If $\mathcal{F}$ is a coherent $\mathcal{O}_{X}$-module, then the degree of $\mathcal{F}$ with respect to $\omega_{X}$ is defined as

$$
\operatorname{deg} \mathcal{F}:=\int_{X} c_{1}(\mathcal{F}) \wedge \omega^{n-1} .
$$

We denote by $E$ a holomorphic vector bundle over $X$ of rank $r$.

Definition 2.1. (i) A Higgs field on a vector bundle $E$ over $X$ is a holomorphic section

$$
\varphi \in H^{0}\left(X, \operatorname{End}(E) \otimes_{\mathcal{O}_{X}} \Omega_{X}^{1}\right)
$$

such that

$$
\varphi \wedge \varphi=0
$$

i.e., $\left[\varphi_{\alpha}, \varphi_{\gamma}\right]=0$ for all $\alpha, \gamma$, where $\varphi=\sum_{\alpha=1}^{n} \varphi_{\alpha} d z^{\alpha}$.

(ii) A Higgs bundle is a pair $(E, \varphi)$, where $\varphi$ is a Higgs field on $E$.

The definition of stability in this context is the following:

Definition 2.2. (i) A Higgs bundle $(E, \varphi)$ is called stable, if

$$
\frac{\operatorname{deg} \mathcal{F}}{\operatorname{rk} \mathcal{F}}<\frac{\operatorname{deg} E}{\operatorname{rk} E}
$$

for all $\mathcal{O}_{X}$-coherent subsheaves $\mathcal{F}$ of $E$ satisfying the conditions $\varphi(\mathcal{F}) \subset$ $\mathcal{F} \otimes_{\mathcal{O}_{X}} \Omega_{X}^{1}$ and $0<\mathrm{rk} \mathcal{F}<\mathrm{rk} E$.

(ii) A polystable Higgs bundle is a direct sum of stable Higgs bundles $\left(E^{\nu}, \varphi^{\nu}\right)$ with the same quotient $\frac{\operatorname{deg} E^{\nu}}{\mathrm{rk} E^{\nu}}$.

Polystable Higgs bundles $(E, \varphi)$ are known to carry a unique HermitianYang-Mills connection by results of Hitchin and Simpson [1,2]; see also [11]. 
Definition 2.3. Let $(E, \varphi)$ be a Higgs bundle. A Hermitian-Yang-Mills connection on $(E, \varphi)$ is a Hermitian connection $\theta_{E}$ on $E$ with curvature form $\Omega_{E}$ such that

$$
\Lambda\left(\Omega_{E}+\varphi \wedge \varphi^{*}\right)=\lambda \cdot \operatorname{id}_{E}
$$

for some $\lambda \in \mathbb{R}$, where $\Lambda=\Lambda_{X}$ is the adjoint to the exterior multiplication of a form with $\omega_{X}$. In local holomorphic coordinates $z^{\alpha}$, this equation reads

$$
g^{\bar{\beta} \alpha}\left(R_{\alpha \bar{\beta}}+\left[\varphi_{\alpha}, \varphi_{\bar{\beta}}^{*}\right]\right)=\lambda \cdot \operatorname{id}_{E},
$$

where $\Omega_{E}=R_{\alpha \bar{\beta}} d z^{\alpha} \wedge d z^{\bar{\beta}}$.

General theory provides a semi-universal deformation of pairs $(E, \varphi)$, where $\varphi$ is a $\operatorname{End}(E)$-valued holomorphic 1-form. The integrability condition $\varphi \wedge \varphi=0$ defines a complex analytic subspace of the parameter space and thus yields a semi-universal deformation for Higgs bundles. It follows like in the classical case that stable Higgs bundles are simple in the sense

$$
H^{0}(X, \operatorname{End}(E, \varphi))=\mathbb{C} \cdot \operatorname{id}_{E},
$$

where

$$
\operatorname{End}(E, \varphi) \subset \operatorname{End}(E)
$$

is the subsheaf that commutes with $\varphi$. A holomorphic family $\left(E_{s}, \varphi_{s}\right)_{s \in S}$ of Higgs bundles, parameterized by a complex space $S$, consists of a holomorphic vector bundle $\mathcal{E}$ on $X \times S$ and a holomorphic section $\Phi$ of $\operatorname{End}(\mathcal{E}) \otimes$ $\rho^{*} \Omega_{X}^{1}$, where $\rho: X \times S \rightarrow X$ is the canonical projection, such that $\mathcal{E} \mid X \times$ $\{s\}=E_{s}$, and $\Phi \mid X \times\{s\}=\varphi_{s}$ for all $s \in S$.

Observe that $\Phi$ defines an $\operatorname{End}(E)$-valued, holomorphic 1-form on $X \times$ $S$, as $\rho^{*} \Omega_{X}^{1} \subset \Omega_{X \times S}^{1}$.

Let $(E, \varphi)$ be any stable Higgs bundle. In a local holomorphic family of Higgs bundles over a pointed space $\left(S, s_{0}\right)$ with $(E, \varphi)$ as the central fiber (the Higgs bundle over $s_{0}$ ), any isomorphism of the central fiber can be extended to the restriction of the family over a neighborhood of $s_{0}$. So, stable Higgs bundles possess universal deformations by general deformation theory [6].

As the uniquely determined Hermitian-Yang-Mills connections on stable Higgs bundles depend in a $C^{\infty}$ way on the parameter of a holomorphic family 
of such bundles, again by general results (even in the non-reduced category) a coarse moduli space exists [6].

We will denote by $\mathcal{M}_{\mathrm{H}}$ a moduli space of stable Higgs bundles over $X$.

We will use the following conventions. The Kähler form $\omega_{X}$ gives rise to a connection on $X$, which we will extend in a flat way to $X \times S$. As above, we will denote by $z^{\alpha}, z^{\gamma}, \ldots$ local coordinates on $X$ together with the conjugates $z^{\bar{\beta}}, z^{\bar{\delta}}, \ldots$ and by $s^{i}, s^{k}, \ldots$ and $s^{\bar{j}}, s^{\bar{\ell}}, \ldots$, respectively, similar coordinates on $S$. We use the semi-colon notation for covariant derivatives of sections and differential forms or tensors with values in the respective vector bundles induced by the Kähler metric on $X$ and the Hermitian connection on the bundle. Let the Hermitian connection $\theta_{E}$ on $E$ be given locally by $\operatorname{End}(E)$-valued $(1,0)$-forms $\left\{\theta_{\alpha}\right\}_{\alpha=1}^{n}$ with respect to some local trivialization of $E$. Let $\sigma$ be a locally defined section of $\operatorname{End}(E)$, which is a matrix-valued function with respect to the trivialization of $E$. We use

$$
\frac{\partial \sigma}{\partial z^{\alpha}}=\partial_{\alpha} \sigma=\sigma_{\mid \alpha}
$$

and set

$$
\nabla_{\alpha} \sigma=\sigma_{; \alpha}=\sigma_{\mid \alpha}+\left[\sigma, \theta_{\alpha}\right]
$$

and

$$
\sigma_{; \bar{\beta}}=\sigma_{\mid \bar{\beta}}
$$

Hence,

$$
\sigma_{; \alpha \bar{\beta}}=\sigma_{; \bar{\beta} \alpha}+\left[\sigma, R_{\alpha \bar{\beta}}\right]
$$

where $R_{\alpha \bar{\beta}}$ denote the components of the curvature form $\Omega_{\alpha \bar{\beta}}=\theta_{\alpha \mid \bar{\beta}}$. For tensors with values in the endomorphism bundle, we also have the contributions that arise from the Kähler connection on the base. We denote by $g d V$ the volume element $\omega_{X}^{n} / n$ ! of the given Kähler form.

\section{Infinitesimal deformations of Higgs bundles}

Let $(E, \varphi)$ be any Higgs bundle over the compact Kähler manifold $X$. For any integer $i \geq 0$, the Higgs field $\varphi$ gives a $\mathcal{O}_{X}$-linear homomorphism

$$
f_{\varphi}(i): \operatorname{End}(E) \otimes \Omega_{X}^{i} \longrightarrow \operatorname{End}(E) \otimes \Omega_{X}^{i+1}
$$

defined by $s \longmapsto[s, \varphi]$. From the given condition that $\varphi \wedge \varphi=0$, it follows immediately that

$$
f_{\varphi}(i+1) \circ f_{\varphi}(i)=0
$$


for all $i$. In other words, there is a complex of $\mathcal{O}_{X}$-coherent modules

$$
\begin{aligned}
D^{\bullet}: 0 \rightarrow D^{0}: & \operatorname{End}(E) \stackrel{f_{\varphi}(0)}{\longrightarrow} \operatorname{End}(E) \otimes \Omega_{X}^{1} \stackrel{f_{\varphi}(1)}{\longrightarrow} \ldots \stackrel{f_{\varphi}(i-1)}{\longrightarrow} \\
D^{i}:= & \operatorname{End}(E) \otimes \Omega_{X}^{i} \stackrel{f_{\varphi}(i)}{\longrightarrow} \operatorname{End}(E) \otimes \Omega_{X}^{i+1} \stackrel{f_{\varphi}(i+1)}{\longrightarrow} \ldots \stackrel{f_{\varphi}(n-1)}{\longrightarrow} \\
& \times \operatorname{End}(E) \otimes \Omega_{X}^{n} \longrightarrow 0
\end{aligned}
$$

over $X$. We note that $H^{0}(X, \operatorname{End}(E, \varphi))=\mathbb{H}^{0}\left(D^{\bullet}\right)$ (see $\left.(2.4)\right)$. The space of all infinitesimal deformations of $(E, \varphi)$ is parameterized by the first hypercohomology $\mathbb{H}^{1}\left(D^{\bullet}\right)$, and the obstructions to deformations of $(E, \varphi)$ are guided by $\mathbb{H}^{2}\left(D^{\bullet}\right)$; see [7] for the details.

For computational convenience, we will work with the Dolbeault resolution of the above complex $D^{\bullet}$.

Consider the spaces

$$
C^{p, q}:=\mathcal{A}^{p, q}(X, \operatorname{End}(E))
$$

of differentiable $(p, q)$-forms over $X$, with values in $\operatorname{End}(E)$ equipped with the Dolbeault operator

$$
d^{\prime \prime}: C^{p, q} \rightarrow C^{p, q+1}
$$

which is the $\bar{\partial}$-operator on $\operatorname{End}(E)$-valued forms. We also have an operator

$$
d^{\prime}: C^{p, q} \rightarrow C^{p+1, q}
$$

which is defined by

$$
d^{\prime}(\chi)=[\chi, \varphi]
$$

Here, the Lie bracket operation sends

$$
\chi_{\gamma \bar{\delta}} d z^{\gamma} \wedge d z^{\bar{\delta}}=\chi_{\gamma_{1}, \ldots, \gamma_{p}, \bar{\delta}_{1}, \ldots, \bar{\delta}_{q}} d z^{\gamma_{1}} \wedge \cdots \wedge d z^{\gamma_{p}} \wedge d z^{\bar{\delta}_{1}} \wedge \cdots \wedge d z^{\bar{\delta}_{q}}
$$

to

$$
[\chi, \varphi]=\left[\chi_{\gamma \bar{\delta}}, \varphi_{\alpha}\right] d z^{\gamma} \wedge d z^{\bar{\delta}} \wedge d z^{\alpha}
$$

Since the section $\varphi$ is holomorphic with $\varphi \wedge \varphi=0$, it follows that $\left(C^{\bullet \bullet}, d^{\prime}, d^{\prime \prime}\right)$ is actually a double complex. This double complex gives rise to a degenerating spectral sequence, which converges to the hypercohomology of the complex $D^{\bullet}$ defined earlier.

For the induced single complex $\left(C^{\bullet}, d\right)$ with

$$
C^{r}:=\bigoplus_{p+q=r} C^{p, q}
$$


we use the homomorphism

$$
d:=d^{\prime \prime}+(-1)^{q+1} d^{\prime}
$$

The groups $\mathbb{H}^{q}\left(C^{\bullet \bullet}\right):=H^{q}\left(C^{\bullet}\right)$, for $q=0,1$, are computed from the truncated complex

$$
0 \longrightarrow C^{0,0} \stackrel{d^{0}}{\longrightarrow} C^{1,0} \oplus C^{0,1} \stackrel{d^{1}}{\longrightarrow} C^{2,0} \oplus C^{1,1} \oplus C^{0,2},
$$

where

$$
d^{0}(f)=(-[f, \varphi], \bar{\partial} f)
$$

and

$$
d^{1}(a, b)=(-[a, \varphi], \bar{\partial} a+[b, \varphi], \bar{\partial} b)
$$

are defined above.

Lemma 3.1. Assume that $(E, \varphi)$ is equipped with a Hermitian-Yang-Mills connection (see Definition 2.3). Then, any holomorphic section of $\operatorname{End}(E, \varphi)$ (defined in (2.4)) is parallel with respect to the induced connection.

Proof. Let $\sigma$ be a holomorphic section of $\operatorname{End}(E, \varphi)$. From $(2.2)$,

$$
g^{\bar{\beta} \alpha}\left(\left[\sigma, R_{\alpha \bar{\beta}}\right]+\left[\sigma,\left[\varphi_{\alpha}, \varphi_{\bar{\beta}}^{*}\right]\right]\right)=0 .
$$

Hence,

$$
\begin{aligned}
\int_{X} g^{\bar{\beta} \alpha} \operatorname{tr} \sigma_{; \alpha} \sigma_{; \bar{\beta}}^{*} g d V & =-\int_{X} g^{\bar{\beta} \alpha} \operatorname{tr} \sigma_{; \alpha \bar{\beta}} \sigma^{*} g d V \\
& =-\int_{X} g^{\bar{\beta} \alpha} \operatorname{tr}\left[\sigma, R_{\alpha \bar{\beta}}\right] \sigma^{*} g d V .
\end{aligned}
$$

Now $\left[\sigma, \varphi_{\alpha}\right]=0$ implies that the above integral equals

$$
\begin{aligned}
-\int_{X} g^{\bar{\beta} \alpha} \operatorname{tr}\left[\varphi_{\alpha},\left[\varphi_{\bar{\beta}}^{*}, \sigma\right]\right] \sigma^{*} g d V & =-\int_{X} g^{\bar{\beta} \alpha} \operatorname{tr} \sigma^{*}\left[\varphi_{\alpha},\left[\varphi_{\bar{\beta}}^{*}, \sigma\right]\right] g d V \\
& =-\int_{X} g^{\bar{\beta} \alpha} \operatorname{tr}\left[\sigma^{*}, \varphi_{\alpha}\right]\left[\varphi_{\bar{\beta}}^{*}, \sigma\right] g d V . \\
& \leq 0 .
\end{aligned}
$$

So the integral vanishes, and $\sigma_{; \alpha}=0$. 
From now on, we assume that the given Higgs bundle $(E, \varphi)$ is stable. Therefore, it carries a Hermitian metric satisfying the Hermitian-Yang-Mills equation (see Definition 2.3). This metric is unique up to a dilation by a globally constant scalar.

Obviously, the space $H^{0}\left(C^{\bullet}\right)$ consists of those holomorphic sections of $\operatorname{End}(E)$ which commute with $\varphi$. The stability condition of $(E, \varphi)$ implies that any such section is a constant scalar multiple of the identity automorphism of $E$ (see (2.3)).

As mentioned earlier, the hypercohomology $\mathbb{H}^{1}\left(D^{\bullet}\right)=\mathbb{H}^{1}\left(C^{\bullet \bullet}\right)=$ $H^{1}\left(C^{\bullet}\right)$ is the space of all infinitesimal deformations of $(E, \varphi)$. We denote the Kodaira-Spencer map by

$$
\rho: T_{s_{0}} S \rightarrow \mathbb{H}^{1}\left(C^{\bullet \bullet}\right)
$$

Now $\left(C^{\bullet}, d\right)$ becomes an elliptic complex, when equipped with the inner products induced by the Hermitian metric on $E$ and the Kähler metric $\omega_{X}$ on $X$. In particular, the formal adjoint operators to $d^{r}$ are in fact adjoint.

More precisely, let $\sigma, \tau \in C^{0,0}$, then

$$
\langle\sigma, \tau\rangle=\int_{X} \operatorname{tr}\left(\sigma \tau^{*}\right) g d V
$$

where $\tau^{*}$ denotes the adjoint section with respect to the Hermitian metric on $E$. For $\operatorname{End}(E)$-valued $(1,0)$-forms $\varphi=\varphi_{\alpha} d z^{\alpha}$ and $\psi=\psi_{\alpha} d z^{\alpha}$, we get

$$
\langle\varphi, \psi\rangle=\int_{X} g^{\bar{\beta} \alpha} \operatorname{tr} \varphi_{\alpha} \psi_{\bar{\beta}}^{*} g d V
$$

As usual, $\Lambda$ denotes the operator that is adjoint to the exterior multiplication of a form with $\omega_{X}$. We mention we have $[a, b]^{*}=-\left[a^{*}, b^{*}\right]$ for any forms $a, b \in C^{p, q}$.

For the computation of adjoint derivatives, we need the following notation. For $v \in C^{1,1}$, we consider $\left[v, \varphi^{*}\right]$ as a tensor rather than as an alternating form. Then,

$$
\widetilde{\Lambda}\left[v_{\alpha \bar{\beta}}, \varphi_{\bar{\delta}}^{*}\right]:=-g^{\bar{\delta} \alpha}\left[v_{\alpha \bar{\beta}}, \varphi_{\bar{\delta}}^{*}\right] d z^{\bar{\beta}} .
$$

So the contraction $\widetilde{\Lambda}\left[v, \varphi^{*}\right]$ stands for the contraction of $v$ and $\varphi^{*}$, and it does not comprise $\Lambda v$. 
Lemma 3.2. For $(a, b) \in C^{1,0} \oplus C^{0,1}$ and $(u, v, w) \in C^{2,0} \oplus C^{1,1} \oplus C^{0,2}$, the following hold:

$$
\begin{gathered}
d^{0 *}(a, b)=-\Lambda\left[a, \varphi^{*}\right]+\bar{\partial}^{*} b \\
d^{1 *}(u, v, w)=\left(\Lambda\left[u, \varphi^{*}\right]+\bar{\partial}^{*} v, \widetilde{\Lambda}\left[v, \varphi^{*}\right]+\bar{\partial}^{*} w\right),
\end{gathered}
$$

where $d^{0}$ and $d^{1}$ are defined in (3.3) and (3.4), respectively.

Proof. The first equation follows from $\langle(a, b), d f\rangle=-\langle a,[f, \varphi]\rangle+\left\langle\bar{\partial}^{*} b, f\right\rangle$ and

$$
-\langle a,[f, \varphi]\rangle=\langle a,[f, \varphi]\rangle=-\left\langle\Lambda\left[a, \varphi^{*}\right], f\right\rangle
$$

whereas for (3.7), we have

$$
\begin{aligned}
& \langle(u, v, w),(-[a, \varphi], \bar{\partial} a+[b, \varphi], \bar{\partial} b)\rangle \\
& \quad=\left\langle-\Lambda\left[u, \varphi^{*}\right], a\right\rangle+\left\langle\bar{\partial}^{*} v, a\right\rangle+\left\langle-\widetilde{\Lambda}\left[v, \varphi^{*}\right], b\right\rangle+\left\langle\bar{\partial}^{*} v, a\right\rangle
\end{aligned}
$$

finishing the proof of the lemma.

Let $(\mathcal{E}, \Phi)$ be a holomorphic family of Higgs bundles over a complex space $S$, and denote by $\left\{h_{s}\right\}$ any $C^{\infty}$ family of Hermitian metrics on $\mathcal{E}_{s}$, i.e., a Hermitian metric $h$ on $\mathcal{E}$ over $X \times S$. Let $\left(s^{1}, \ldots, s^{k}\right)$ be holomorphic coordinates on $S$, if $S$ is smooth, or holomorphic coordinates on an ambient smooth space into which a neighborhood of $s_{0} \in S$ is minimally embedded.

Let $\Omega$ be the curvature form of the Hermitian connection for $h$ on $\mathcal{E}$ over $X \times S$. The curvature tensor for this connection will be denoted by $R$. So the contraction

$$
\Omega\left\llcorner\frac{\partial}{\partial s_{i}}\right.
$$

equals

$$
R_{i \bar{\beta}} d z^{\bar{\beta}}
$$

Following the construction in [4], one can see that the global tensors $\Omega$ and $\varphi$ over $X \times S$ already describe the infinitesimal deformations. In other words, we have the following lemma: 
Lemma 3.3. The Kodaira-Spencer class

$$
\rho\left(\left.\frac{\partial}{\partial s_{i}}\right|_{s_{0}}\right) \in \mathbb{H}^{1}\left(C^{\bullet \bullet}\right)
$$

(the homomorphism $\rho$ is defined in (3.5)) is represented by

$$
\eta_{i}=\left.\left(\varphi_{\alpha ; i} d z^{\alpha}, R_{i \bar{\beta}} d z^{\bar{\beta}}\right)\right|_{X \times\left\{s_{0}\right\}} \cdot
$$

The Kähler form $\omega_{X}$ and the Hermitian metric $h$ together provide the above double complex $C^{\bullet \bullet}$ with a natural inner product such that the adjoint operators $d^{j *}$ are the formal adjoint operators.

Now assume that for each point $s \in S$, the Higgs bundle $\left(\mathcal{E}_{s}, \Phi_{s}\right)$ over $X$ is stable. The Hermitian-Yang-Mills connections on this family of stable Higgs bundles $(\mathcal{E}, \Phi)$ are induced by a Hermitian metric $h$ on $\mathcal{E}$, whose curvature form $\Omega$ is unique up to a differential form of type $\operatorname{id}_{E} \otimes f^{*} \omega^{\prime}$, where $\omega^{\prime}$ is some $(1,1)$-form on the base $S$ and $f: X \times S \rightarrow S$ is the natural projection. Indeed, this follows immediately from the fact that any two Hermitian-Yang-Mills metrics on a stable Higgs bundle differ by multiplication with a constant scalar.

Therefore, the components $R_{i \bar{\beta}}$ of the curvature tensor in Lemma 3.3 are uniquely determined by the family of Hermitian-Yang-Mills connections on the Higgs bundles $\left(\mathcal{E}_{s}, \varphi_{s}\right)$.

Proposition 3.4. The $\operatorname{End}(\mathcal{E})$-valued 1 -forms

$$
\eta_{i}=\varphi_{\alpha ; i} d z^{\alpha}+R_{i \bar{\beta}} d z^{\bar{\beta}}
$$

are the harmonic representatives of the Kodaira-Spencer classes $\rho\left(\partial /\left.\partial s^{i}\right|_{s_{0}}\right)$.

Proof. Following Lemma 3.2, we find

$$
\begin{aligned}
d^{*}\left(\varphi_{\alpha ; i} d z^{\alpha}, R_{i \bar{\beta}} d z^{\bar{\beta}}\right) & =-\Lambda\left[\varphi_{; i}, \varphi^{*}\right]+\bar{\partial}^{*}\left(R_{i \bar{\beta}} d z^{\bar{\beta}}\right) \\
& =g^{\bar{\beta} \alpha}\left(-\left[\varphi_{\alpha ; i}, \varphi_{\bar{\beta}}^{*}\right]-R_{\alpha \bar{\beta} ; i}\right)=0
\end{aligned}
$$

because of the Hermitian-Yang-Mills condition (2.2) for Higgs bundles.

For applications in Section 8, we introduce a decomposition of the complex $D^{\bullet}$. 
Let

$$
\operatorname{ad}(E) \subset \operatorname{End}(E)
$$

be the subbundle of trace zero endomorphisms, and let

$$
\text { pr: } \operatorname{End}(E) \longrightarrow \operatorname{ad}(E)
$$

defined by $\operatorname{pr}(\chi):=\chi-(1 / \operatorname{rk}(E)) \operatorname{tr}(\chi) \operatorname{id}_{E}$ be the projection onto the tracefree part. We extend the homomorphism pr to the complex $D^{\bullet}$ and its resolution. Now the complex of $\mathcal{O}_{X}$-coherent modules

$$
\begin{aligned}
D_{0}^{\bullet}: 0 \longrightarrow D_{0}^{0}:= & \operatorname{ad}(E) \stackrel{f_{\varphi}(0)}{\longrightarrow} \operatorname{ad}(E) \otimes \Omega_{X}^{1} \stackrel{f_{\varphi}(1)}{\longrightarrow} \ldots \stackrel{f_{\varphi}(i-1)}{\longrightarrow} D_{0}^{i} \\
:= & \operatorname{ad}(E) \otimes \Omega_{X}^{i} \stackrel{f_{\varphi}(i)}{\longrightarrow} \operatorname{ad}(E) \otimes \Omega_{X}^{i+1} \stackrel{f_{\varphi}(i+1)}{\longrightarrow} \ldots \stackrel{f_{\varphi}(n-1)}{\longrightarrow} \\
& \times \operatorname{ad}(E) \otimes \Omega_{X}^{n} \longrightarrow 0
\end{aligned}
$$

over $X$ is a subcomplex of the complex $D^{\bullet}$.

We identify $\Omega_{X}^{k}$ with $\operatorname{id}_{E} \otimes \Omega_{X}^{i} \subset D^{i}$.

Lemma 3.5. The restrictions of the chain morphisms $d^{k}$ to $\Omega_{X}^{k}$ are identically zero. Moreover,

$$
D^{\bullet}=D_{0}^{\bullet} \oplus \Omega_{X}^{\bullet}
$$

is an orthogonal decomposition. The resolution of $C^{\bullet \bullet}$ also decomposes in an orthogonal way

$$
C^{\bullet \bullet}=C_{0}^{\bullet \bullet} \oplus \mathcal{A}_{X}^{\bullet \bullet},
$$

where $\mathcal{A}_{X}^{\bullet \bullet}$ corresponds to the Dolbeault resolution of $\Omega_{X}^{\bullet}$. In particular, the following holds.

The subcomplex $C_{0}^{\bullet \bullet} \subset C^{\bullet \bullet}$ is preserved by both $d_{0}$ and $d_{0}^{*}$. Let $d_{0}$ and $d_{0}^{*}$ be the restrictions of $d$ and $d^{*}$, respectively, to $C_{0}^{\bullet \bullet}$. Then,

$$
\text { prod }=d_{0} \circ \text { pr } \quad \text { and } \quad \text { pro } d^{*}=d_{0}^{*} \circ \text { pr. }
$$

Proof. The proof follows from $\operatorname{tr}[\chi, \varphi]=0$ for any $\chi \in D^{k}$ and the simple fact that covariant derivatives commute with taking traces.

Concerning the second cohomology, we note

Lemma 3.6. There is a natural embedding

$$
\mathbb{C} \cdot \omega_{X} \cdot \operatorname{id}_{E} \hookrightarrow \mathbb{H}^{2}\left(C^{\bullet \bullet}\right) .
$$


Proof. We have to consider

$$
\epsilon=\left(0, \omega_{X} \cdot \operatorname{id}_{E}, 0\right) \in C^{2,0} \oplus C^{1,1} \oplus C^{0,2} .
$$

Then, $d \epsilon=\left(0,\left[\omega_{X} \cdot \operatorname{id}_{E}, \varphi\right], \bar{\partial}\left(\omega_{X} \cdot \operatorname{id}_{E}\right), 0\right)=0$, and from $(3.7)$,

$$
d^{*} \epsilon=\left(\bar{\partial}^{*}\left(\omega_{X} \cdot \operatorname{id}_{E}\right), \widetilde{\Lambda}\left[\omega_{X} \cdot \operatorname{id}_{E}, \varphi^{*}\right]\right)=0 .
$$

So $\epsilon$ is harmonic, and it is different from zero, since $\int_{X} \operatorname{tr} \epsilon^{n} \neq 0$.

We will need the following proposition.

Proposition 3.7. The obstructions for deformations of a Higgs bundle $(E, \varphi)$ are already contained in the second hypercohomology $\mathbb{H}^{2}\left(D_{0}^{\bullet}\right)$. If $\operatorname{dim} \mathbb{H}^{2}\left(D^{\bullet}\right)=1$, then we have $\mathbb{H}^{2}\left(D_{0}^{\bullet}\right)=0$.

Proof. Note that the deformations of any Higgs line bundle are unobstructed (as the deformations of a line bundle are so). Therefore, setting $G=\operatorname{PGL}(r, \mathbb{C})$ in Theorem 3.1 of [8], where $r=\operatorname{rank}(E)$, we conclude that if the image of $\mathbb{H}^{2}\left(D^{\bullet}\right)$ in $\mathbb{H}^{2}\left(D_{0}^{\bullet}\right)$ is zero, then all deformations of $(E, \varphi)$ are unobstructed.

If $\operatorname{dim} \mathbb{H}^{2}\left(D^{\bullet}\right)=1$, then $\mathbb{H}^{2}\left(D^{\bullet}\right)$ must be generated by the image of the non-zero homomorphism in Lemma 3.6, and hence $\mathbb{H}^{2}\left(D_{0}^{\bullet}\right)$ vanishes.

\section{Generalized Petersson-Weil metric}

Now, we are in a position to introduce a generalized Petersson-Weil metric on the parameter space $S$ for a family of stable Higgs bundles. The generalized Petersson-Weil metric is an inner product $G^{\mathrm{PW}}$ on the tangent spaces $T_{s} S$ of the bases of holomorphic families, which is positive definite for effective families, and it is defined in terms of the tensors $\eta_{i}$ representing the Kodaira-Spencer classes. This is possible, also in the case where $S$ is singular, because the family of Higgs forms, and the curvature form for the connection on vector bundles, still exists on the first infinitesimal neighborhood. The latter fact follows from the approach that is based on the implicit function theorem.

We call this Hermitian structure the generalized Petersson-Weil metric and use the following notation:

$$
\begin{aligned}
G^{\mathrm{PW}} & \left(\left.\frac{\partial}{\partial s^{i}}\right|_{s_{0}},\left.\frac{\partial}{\partial s^{\bar{\jmath}}}\right|_{s_{0}}\right):=G_{i \bar{\jmath}}^{\mathrm{PW}}:=\left\langle\eta_{i}, \eta_{j}\right\rangle \\
& =\int_{X} \operatorname{tr}\left(g^{\bar{\beta} \alpha} \varphi_{\alpha ; i} \varphi_{\bar{\beta}, \bar{\jmath}}^{*}\right) g d V+\int_{X} \operatorname{tr}\left(g^{\bar{\beta} \alpha} R_{i \bar{\beta}} R_{\alpha \bar{\jmath}}\right) g d V .
\end{aligned}
$$


We set

$$
\omega_{\mathrm{PW}}=\sqrt{-1} G_{i \bar{\jmath}}^{\mathrm{PW}} d s^{i} \wedge d s^{\bar{\jmath}} .
$$

In order to compute the induced connection, we need certain identities.

Lemma 4.1. Let $\eta_{i}=\left(\varphi_{\alpha ; i} d z^{\alpha}, R_{i \bar{\beta}} d z^{\bar{\beta}}\right)$. Then,

$$
\begin{gathered}
\eta_{i ; k}=\eta_{k ; i} \\
d \eta_{i ; k}+\eta_{i} \wedge \eta_{k}=0 \\
d^{*} \eta_{i ; k}=0 \\
\eta_{i ; \bar{\jmath}}=d R_{i \bar{\jmath}} \\
\square R_{i \bar{\jmath}}=d^{*} d R_{i \bar{\jmath}}=d^{*} \eta_{i ; \bar{\jmath}} \\
d^{*} \eta_{i ; \bar{\jmath}}=g^{\bar{\beta} \alpha}\left(\left[\varphi_{\alpha ; i}, \varphi_{\bar{\beta}, \bar{\jmath}}^{*}\right]+\left[R_{i \bar{\beta}}, R_{\alpha \bar{\jmath}}\right]\right) .
\end{gathered}
$$

Proof. The symmetry (4.2) of $\eta_{i ; k}$ follows immediately from

$$
\eta_{i ; k}=\left(\varphi_{\alpha ; i k} d z^{\alpha}, R_{i \bar{\beta} ; k} d z^{\bar{\beta}}\right),
$$

which is symmetric in $i$ and $k$.

We show (4.3):

$$
\begin{aligned}
d \eta_{i ; k}= & \left(-\left[\varphi_{\alpha ; i k}, \varphi_{\gamma}\right] d z^{\alpha} \wedge d z^{\gamma}, \varphi_{\alpha ; i k \bar{\beta}} d z^{\bar{\beta}} \wedge d z^{\alpha}\right. \\
& \left.+\left[R_{i \bar{\beta} ; k} d z^{\bar{\beta}}, \varphi_{\alpha} d z^{\alpha}\right], R_{i \bar{\beta} ; k \bar{\delta}} d z^{\bar{\delta}} \wedge d z^{\bar{\beta}}\right) \\
= & \left(\left[\varphi_{; i}, \varphi_{; k}\right],-\left[\varphi_{; i}, R_{k \bar{\beta}} d z^{\bar{\beta}}\right]-\left[\varphi_{; k}, R_{i \bar{\beta}} d z^{\bar{\beta}}\right],-\left[R_{i \bar{\beta}} d z^{\bar{\beta}}, R_{k \bar{\delta}} d z^{\bar{\delta}}\right]\right) .
\end{aligned}
$$

This gives the formula (with the given sign convention for $C^{\bullet \bullet}$ ).

Concerning (4.4), we have

$$
d^{*} \eta_{i ; k}=-g^{\bar{\beta} \alpha}\left[\varphi_{\alpha ; i k}, \varphi_{\bar{\beta}}^{*}\right]-g^{\bar{\beta} \alpha} R_{i \bar{\beta} ; k \alpha} .
$$

We use (2.2) in

$$
\begin{aligned}
-g^{\bar{\beta} \alpha} R_{i \bar{\beta} ; k \alpha} & =-g^{\bar{\beta} \alpha} R_{\alpha \bar{\beta} ; i k}=g^{\bar{\beta} \alpha}\left[\varphi_{\alpha}, \varphi_{\bar{\beta}}^{*}\right]_{; i k} \\
& =g^{\bar{\beta} \alpha}\left[\varphi_{\alpha ; i}, \varphi_{\bar{\beta}}^{*}\right]_{; k}=g^{\bar{\beta} \alpha}\left[\varphi_{\alpha ; i k}, \varphi \frac{*}{\beta}\right] .
\end{aligned}
$$

Next, we show (4.5),

$$
\begin{aligned}
\eta_{i ; \bar{\jmath}} & =\left(\varphi_{\alpha ; i} d z^{\alpha}, R_{i \bar{\beta}} d z^{\bar{\beta}}\right)_{; \bar{\jmath}}=\left(\left(\varphi_{\alpha ; \bar{\jmath} i}+\left[\varphi_{\alpha}, R_{i \bar{\jmath}}\right]\right) d z^{\alpha}, R_{i \bar{\jmath} ; \bar{\beta}} d z^{\bar{\beta}}\right) \\
& =\left(-\left[R_{i \bar{\jmath}}, \varphi\right], \bar{\partial} R_{i \jmath}\right)=d R_{i \bar{\jmath}} .
\end{aligned}
$$


Formula (4.6) is immediate. For the last formula (4.7), we consider

$$
\begin{aligned}
d^{*} \eta_{i ; \bar{\jmath}} & =g^{\bar{\beta} \alpha}\left(\left[\varphi_{\alpha ; i \bar{\jmath}}, \varphi_{\bar{\beta}}^{*}\right]-R_{i \bar{\beta} ; \bar{\jmath} \alpha}\right) \\
& =-g^{\bar{\beta} \alpha}\left(\left[\varphi_{\alpha ; \bar{\jmath} i}+\left[\varphi_{\alpha}, R_{i \bar{\jmath}}\right], \varphi_{\bar{\beta}}^{*}\right]+\left(R_{i \bar{\beta} ; \alpha \bar{\jmath}}-\left[R_{i \bar{\beta}}, R_{\alpha \bar{\jmath}}\right]\right)\right) .
\end{aligned}
$$

Using the Hermitian-Yang-Mills equation, we get

$$
\begin{aligned}
& g^{\bar{\beta} \alpha}\left(-\left[\left[\varphi_{\alpha}, R_{i \bar{\jmath}}\right], \varphi_{\bar{\beta}}^{*}\right]+\left[\varphi_{\alpha ; i \bar{\jmath}}, \varphi_{\bar{\beta}}^{*}\right]+\left[\varphi_{\alpha ; i}, \varphi_{\bar{\beta} ; \bar{\jmath}}^{*}\right]+\left[R_{i \bar{\beta}}, R_{\alpha \bar{\jmath}}\right]\right) \\
& \quad=g^{\bar{\beta} \alpha}\left(\left[\varphi_{\alpha ; i}, \varphi_{\bar{\beta} ; \bar{\jmath}}^{*}\right]+\left[R_{i \bar{\beta}}, R_{\alpha \bar{\jmath}}\right]\right)
\end{aligned}
$$

completing the proof of the lemma.

As a consequence of Proposition 3.4 and (4.5), we note that

$$
\left\langle\eta_{i}, \eta_{j ; \bar{k}}\right\rangle=\left\langle\eta_{i}, d R_{j \bar{k}}\right\rangle=\left\langle d^{*} \eta_{i}, R_{j \bar{k}}\right\rangle=0 .
$$

Using the above notation, we set

$$
G_{i \bar{\jmath} \mid k}^{\mathrm{PW}}=\partial_{s_{k}} G_{i \bar{\jmath}}^{\mathrm{PW}} .
$$

Proposition 4.2. The generalized Petersson-Weil metric is Kähler. More precisely, we have

$$
G_{i \bar{\jmath} \mid k}^{\mathrm{PW}}=\left\langle\eta_{i ; k}, \eta_{j}\right\rangle
$$

Proof. We use $G_{i \bar{\jmath} \mid k}^{\mathrm{PW}}=\left\langle\eta_{i ; k}, \eta_{j}\right\rangle+\left\langle\eta_{i}, \eta_{j ; \bar{k}}\right\rangle$ and (4.8).

Corollary 4.3. Let $s_{0}$ be some point of the base $S$ of a universal deformation of a Higgs-bundle. Consider normal coordinates $\left\{s^{i}\right\}$ for the PeterssonWeil metric at the base point $s_{0}$. Then, for all $i, k$, the harmonic projections $H\left(\left.\eta_{i ; k}\right|_{s=s_{0}}\right)$ vanish.

Proof. This follows immediately from the fact that the $\eta_{i}$ are harmonic and span the whole space $H^{1}\left(C^{\bullet}\right)$.

\section{Curvature of the generalized Petersson-Weil metric}

Let $S=\left\{\left(s^{1}, \ldots, s^{N}\right)\right\}$ be the smooth base of a universal deformation of a Higgs bundle equipped with a family of Hermitian-Yang-Mills metrics. Let $\eta_{i}=\left(\varphi_{\alpha ; i} d z^{\alpha}, R_{i \bar{\beta}} d z^{\beta}\right)$ be the harmonic representative of the KodairaSpencer class $\rho\left(\partial /\left.\partial s_{i}\right|_{s}\right) \in \mathbb{H}^{1}\left(C^{\bullet \bullet}\right)$. We consider the associated single 
complex $C^{\bullet}$ as an elliptic complex equipped with the Laplacians $\square=d^{*} d+$ $d d^{*}$ acting on $\operatorname{End}(E)$-valued forms in all degrees. This elliptic complex possesses harmonic projections $H$ and Green's operators $G$.

Theorem 5.1. Let $\eta_{i}=\left(\varphi_{\alpha ; i} d z^{\alpha}, R_{i \bar{\beta}} d z^{\bar{\beta}}\right)$ be the elements of a basis of the harmonic Kodaira-Spencer forms, depending on $s \in S$. Then, the curvature tensor of the generalized Petersson-Weil metric equals

$$
\begin{aligned}
R_{i \bar{\jmath} k \bar{\ell}}^{\mathrm{PW}}= & \int_{X} \operatorname{tr}\left(G\left(\Lambda\left(\eta_{i} \wedge \eta_{\bar{\jmath}}^{*}\right)\right) \Lambda\left(\eta_{k} \wedge \eta_{\bar{\ell}}^{*}\right)\right) g d V \\
& +\int_{X} \operatorname{tr}\left(G\left(\Lambda\left(\eta_{i} \wedge \eta_{\bar{\ell}}^{*}\right)\right) \Lambda\left(\eta_{k} \wedge \eta_{\bar{\jmath}}^{*}\right)\right) g d V \\
& +\int_{X} \operatorname{tr}\left(\left[\eta_{i} \wedge \eta_{k}\right] \wedge G\left(\left[\eta_{\bar{\jmath}}^{*} \wedge \eta_{\bar{\ell}}^{*}\right]\right)\right) \frac{\omega_{X}^{n-1}}{(n-1) !}
\end{aligned}
$$

Explicitly, we have

$$
\begin{aligned}
R_{i \bar{\jmath} k \bar{\ell}}^{\mathrm{PW}}= & +\int_{X} \operatorname{tr}\left(R_{i \bar{\jmath}} \square R_{k \bar{\ell}}+R_{i \bar{\ell}} \square R_{k \bar{\jmath}}\right) g d V \\
& +\int_{X} \operatorname{tr}\left(\left[\eta_{i} \wedge \eta_{k}\right] \wedge G\left(\left[\eta_{\bar{\jmath}}^{*} \wedge \eta_{\bar{\ell}}^{*}\right]\right)\right) \frac{\omega_{X}^{n-1}}{(n-1) !} .
\end{aligned}
$$

Here, $\left[\eta_{i} \wedge \eta_{k}\right]$ and $\left[\eta_{i} \wedge \eta_{\bar{j}}\right]$ stand for the exterior product of forms, with values in an endomorphism bundle combined with the Lie product.

Remark 5.2. The first two terms in (5.1) and the first term in (5.2), respectively, are semi-positive. Because of the different order of non-conjugate and conjugate terms in the first and second part of the curvature formula, the last terms of (5.1) and (5.2), respectively, yield semi-negativity.

Proof of Theorem 5.1. We use normal coordinates at a given point of $S$. Then,

$$
-R_{i \bar{\jmath} k \bar{\ell}}^{\mathrm{PW}}=G_{i \bar{\jmath} \mid k \bar{\ell}}^{\mathrm{PW}}=\left\langle\eta_{i ; k \bar{\ell}}, \eta_{j}\right\rangle+\left\langle\eta_{i ; k}, \eta_{j ; \ell}\right\rangle=: A+B .
$$

We compute $A$.

$$
\eta_{i ; k \bar{\ell}}=\left(\varphi_{\alpha ; i k \bar{\ell}} d z^{\alpha}, R_{i \bar{\beta} ; k \bar{\ell}} d z^{\bar{\beta}}\right) \in C^{1,1}
$$


First, we need

$$
\begin{aligned}
\varphi_{\alpha ; i k \bar{\ell}} & =\varphi_{\alpha ; \bar{\ell} k}+\left[\varphi_{\alpha ; i}, R_{k \bar{\ell}}\right] \\
& =\varphi_{\alpha ; \bar{\ell} i k}+\left[\varphi_{\alpha}, R_{i \bar{\ell}}\right]_{; k}+\left[\varphi_{\alpha ; i}, R_{k \bar{\ell}}\right] \\
& =\left[\varphi_{\alpha ; k}, R_{i \bar{\ell}}\right]+\left[\varphi_{\alpha ; i}, R_{k \bar{\ell}}\right]+\left[\varphi_{\alpha}, R_{\bar{i} \bar{i} ; k}\right]
\end{aligned}
$$

so that

$$
\left\langle\varphi_{\alpha ; i k \bar{\ell}}, \varphi_{\gamma ; j}\right\rangle=\int_{X} g^{\bar{\beta} \alpha} \operatorname{tr}\left(\left(\left[\varphi_{\alpha ; k}, R_{i \bar{\ell}}\right]+\left[\varphi_{\alpha ; i}, R_{k \bar{\ell}}\right]+\left[\varphi_{\alpha}, R_{i \bar{\ell} ; k}\right]\right) \varphi_{\bar{\beta} ; \bar{j}}^{*}\right) g d V
$$

and

$$
R_{i \bar{\beta} ; k \bar{\ell}}=R_{i \bar{\ell}, k \bar{\beta}}-\left[R_{i \bar{\ell}}, R_{k \bar{\beta}}\right]+\left[R_{i \bar{\beta}}, R_{k \bar{\ell}}\right]
$$

so that

$$
\begin{aligned}
\int_{X} g^{\bar{\beta} \alpha} \operatorname{tr}\left(R_{i \bar{\ell} ; k \bar{\beta}} R_{\alpha \bar{\jmath}}\right) g d V & =-\int_{X} g^{\bar{\beta} \alpha} \operatorname{tr}\left(R_{i \bar{\ell} ; k} R_{\alpha \bar{\beta} ; \bar{\jmath}}\right) g d V \\
& =\int_{X} g^{\bar{\beta} \alpha} \operatorname{tr}\left(R_{i \bar{\ell} ; k}\left[\varphi_{\alpha}, \varphi_{\bar{\beta} \bar{\jmath}}^{*}\right]\right) g d V \\
& =\int_{X} g^{\bar{\beta} \alpha} \operatorname{tr}\left(\left[R_{i \bar{\ell} ; k}, \varphi_{\alpha}\right] \varphi_{\bar{\beta} \bar{\jmath}}^{*}\right) g d V
\end{aligned}
$$

which is inserted into the expression for

$$
\left\langle R_{i \bar{\beta} ; k \bar{\ell}} d z^{\bar{\beta}}, R_{j \bar{\delta}} d z^{\bar{\delta}}\right\rangle
$$

So far, we have

$$
\begin{aligned}
A= & \int_{X} g^{\bar{\beta} \alpha} \operatorname{tr}\left(R_{i \bar{\ell}}\left(\left[R_{\alpha \bar{\jmath}}, R_{k \bar{\beta}}\right]-\left[\varphi_{\alpha ; k}, \varphi_{\bar{\beta} ; \bar{\jmath}}^{*}\right]\right)\right. \\
& +R_{k \bar{\ell}}\left(\left[R_{\alpha \bar{\jmath}}, R_{i \bar{\beta}}\right]-\left[\varphi_{\alpha ; i}, \varphi_{\bar{\beta} ; \bar{\jmath}}^{*}\right]\right) g d V
\end{aligned}
$$

where $A$ is defined in (5.3).

Now, we compute the $(1,1)$-component of $\eta_{k} \wedge \eta_{\bar{j}}^{*}$ :

$$
-\left(\eta_{k} \wedge \eta_{\bar{\jmath}}^{*}\right)_{(1,1)}=\left(\left[R_{\alpha \bar{\jmath}}, R_{k \bar{\beta}}\right]-\left[\varphi_{\alpha ; k}, \varphi_{\bar{\beta}, \bar{\jmath}}^{*}\right]\right) d z^{\alpha} \wedge d z^{\bar{\beta}}
$$

so that with (4.6) and (4.7),

$$
\Lambda\left(\eta_{i} \wedge \eta_{\bar{\jmath}}^{*}\right)=d^{*} \eta_{i ; \bar{\jmath}}=\square R_{i \bar{\jmath}} .
$$


Hence,

$$
\begin{aligned}
A & =-\int_{X} \operatorname{tr}\left(R_{i \bar{\jmath}} \square R_{k \bar{\ell}}+R_{i \bar{\ell}} \square R_{k \bar{\jmath}}\right) g d V \\
& =-\int_{X} \operatorname{tr}\left(G\left(\Lambda\left(\eta_{i} \wedge \eta_{\bar{\jmath}}^{*}\right)\right) \Lambda\left(\eta_{k} \wedge \eta_{\bar{\ell}}^{*}\right)+G\left(\Lambda\left(\eta_{i} \wedge \eta_{\bar{\ell}}^{*}\right)\right) \Lambda\left(\eta_{k} \wedge \eta_{\bar{\jmath}}^{*}\right)\right) g d V .
\end{aligned}
$$

We compute $B$ defined in (5.3). Since $d^{*} \eta_{i ; k}=0$, and $H\left(\eta_{i ; k}\right)=0$ by (4.4) and Corollary 4.3, we have

$$
\eta_{i ; k}=G d^{*} d \eta_{i ; k}=d^{*} G d \eta_{i ; k}
$$

and

$$
B=\left\langle d \eta_{i ; k}, G d \eta_{j ; \ell}\right\rangle
$$

so that we get the third term of (5.1) using (4.3). This completes the proof of the theorem.

We estimate the holomorphic sectional curvature for $\operatorname{dim} X=1$ :

$$
R_{i \bar{i} i \bar{i}}^{\mathrm{PW}}=2\left\langle d R_{i \bar{i}}, d R_{i \bar{i}}\right\rangle \geq 0 .
$$

with $d=d^{0}$ (see (3.3)). Equality holds only if $d R_{i \bar{j}}=0$, that is, $R_{i \bar{i}}$ is a holomorphic section of $\operatorname{End}(E, \varphi)$; see $(2.4)$ for the definition of $\operatorname{End}(E, \varphi)$. Since $(E, \varphi)$ is stable, any holomorphic section of $\operatorname{End}(E, \varphi)$ is a constant multiple of the identity automorphism of $E$ (see (2.3)).

Therefore, we have the following corollary:

Corollary 5.3. When $\operatorname{dim} X=1$, the holomorphic sectional curvature of the Petersson-Weil metric is non-negative.

\section{Fiber integral formula}

We will show the existence of a local $\partial \bar{\partial}$-potential for the generalized Petersson-Weil metric on a base space $S$ of a universal deformation. We note that this implies the Kähler condition of the Petersson-Weil metric.

We consider a moduli space of stable Higgs bundles $\mathcal{M}_{\mathrm{H}}$. Although, in general, there is no universal holomorphic vector bundle $\mathcal{E}$ globally on $X \times \mathcal{M}_{\mathrm{H}}$, the bundle $\operatorname{End}(\mathcal{E})$ exists in the orbifold sense over all of $X \times \mathcal{M}_{\mathrm{H}}$, since the non-zero scalars act trivially on $\operatorname{End}(\mathcal{E})$. We will furthermore need the highest exterior power $\Lambda^{r} \mathcal{E}$, a tensor power of which also descends to $X \times \mathcal{M}_{\mathrm{H}}$. 
Representing a point $p \in \mathcal{M}_{\mathrm{H}}$ by the isomorphism class of a Higgs bundle $(E, \varphi)$, we find the existence of a global holomorphic 1-form $\Phi \in H^{0}(X \times$ $\left.\mathcal{M}_{\mathrm{H}}, \Omega_{\mathcal{M}_{\mathrm{H}}}^{1}(\operatorname{End}(\mathcal{E}))\right)$. Hence, the function on $\mathcal{M}_{\mathrm{H}}$ defined by

$$
s \mapsto \chi(s)=\int_{X \times\{s\}} g^{\bar{\beta} \alpha} \operatorname{tr}\left(\varphi_{\alpha} \varphi_{\frac{\beta}{\beta}}^{*}\right) g d V
$$

is a function of class $C^{\infty}$ on $\mathcal{M}_{\mathrm{H}}$.

In a similar way, the curvature form $\Omega$ of the Hermitian-Yang-Mills connections is a well-defined $\operatorname{End}(\mathcal{E})$-valued $(1,1)$-form over $X \times \mathcal{M}_{\mathrm{H}}$.

For the results of this section, the base space $S$ can be a complex space (even non-reduced, if necessary). However, in order to simplify the exposition, we assume smoothness.

Given the projection $X \times S \rightarrow S$, where $S$ is also smooth, the pushforward of a $(n+1, n+1)$-form $\Psi$ (defined on $X \times S)$ is a $(1,1)$-form on $S$ given by a fiber integral

$$
\int_{X \times S / S} \Psi \text {, which we also write as } \int_{X \times\{s\}} \Psi \text { or simply } \int_{X} \Psi \text {. }
$$

The reader may consult [12] for fiber integral.

Proposition 6.1. Let $\Omega$ be the curvature form of $(\mathcal{E}, h)$. Then the following fiber integral formula holds:

$$
\begin{aligned}
\omega_{P W}= & \frac{1}{2} \int_{X} \operatorname{tr}(\Omega \wedge \Omega) \wedge \frac{\omega_{X}^{n-1}}{(n-1) !} \\
& +\lambda \int_{X} \operatorname{tr}(\sqrt{-1} \Omega) \wedge \frac{\omega_{X}^{n}}{n !}+\sqrt{-1} \partial \bar{\partial} \frac{1}{2} \int_{X} \operatorname{tr}\left(\varphi \wedge \varphi^{*}\right) \wedge \frac{\omega_{X}^{n-1}}{(n-1) !} .
\end{aligned}
$$

Here $\lambda$ is determined by

$$
\int_{X} \operatorname{tr}(\sqrt{-1} \Omega) \wedge \frac{\omega_{X}^{n-1}}{(n-1) !}=\lambda \int_{X} \frac{\omega_{X}^{n}}{n !}
$$

is independently of $s \in S$ over any connected component of $S$.

Before we prove the proposition, we recall some standard facts. Concerning Chern character forms, we will use the description as

$$
\operatorname{ch}(\mathcal{E}, h)=\sum_{k=0}^{n} \frac{1}{k !} \operatorname{tr}(\underbrace{\frac{\sqrt{-1}}{2 \pi} \Omega \wedge \cdots \wedge \frac{\sqrt{-1}}{2 \pi} \Omega}_{k})
$$


with

$$
\operatorname{ch}_{2}(\mathcal{E}, h)=\frac{1}{2}\left(c_{1}^{2}(\mathcal{E}, h)-2 c_{2}(\mathcal{E}, h)\right)
$$

In terms of Chern character forms and Chern forms, formula (6.1) reads

$$
\begin{aligned}
\frac{1}{4 \pi^{2}} \omega_{\mathrm{PW}}= & -\int_{X} \operatorname{ch}_{2}(\mathcal{E}, h) \wedge \frac{\omega_{X}^{n-1}}{(n-1) !} \\
& +\frac{\lambda}{2 \pi} \int_{X} c_{1}(\mathcal{E}, h) \wedge \frac{\omega_{X}^{n}}{n !}+\frac{\sqrt{-1}}{8 \pi^{2}} \partial \bar{\partial} \int_{X} \operatorname{tr}\left(\varphi \wedge \varphi^{*}\right) \wedge \frac{\omega_{X}^{n-1}}{(n-1) !} .
\end{aligned}
$$

Now we will prove the proposition.

Proof. By definition

$$
\omega_{\mathrm{PW}}=\left(\int \operatorname{tr}\left(R_{i \bar{\beta}} R_{\alpha \bar{\jmath}}\right) g^{\bar{\beta} \alpha} g d V+\int \operatorname{tr}\left(\varphi_{\alpha ; i} \varphi_{\bar{\beta} ; \bar{\jmath}}^{*}\right) g d V\right) \sqrt{-1} d s^{i} \wedge d s^{\bar{\jmath}} .
$$

Now

$$
\begin{aligned}
& \frac{1}{2} \int_{X} \operatorname{tr}(\Omega \wedge \Omega) \wedge \frac{\omega_{X}^{n-1}}{(n-1) !} \\
& \quad=-\frac{1}{2} \int_{X} \operatorname{tr}(\sqrt{-1} \Omega \wedge \sqrt{-1} \Omega) \wedge \frac{\omega_{X}^{n-1}}{(n-1) !} \\
& \quad=\int \operatorname{tr}\left(R_{\alpha \bar{\jmath}} \cdot R_{i \bar{\beta}}-R_{\alpha \bar{\beta}} \cdot R_{i \bar{\jmath}}\right) g^{\bar{\beta} \alpha} g d V \sqrt{-1} d s^{i} \wedge d s^{\bar{\jmath}},
\end{aligned}
$$

and from (2.2), we have

$$
-\operatorname{tr}\left(g^{\bar{\beta} \alpha} R_{\alpha \bar{\beta}} \cdot R_{i \bar{\jmath}}\right)=\operatorname{tr}\left(g^{\bar{\beta} \alpha}\left[\varphi_{\alpha}, \varphi_{\bar{\beta}}^{*}\right] \cdot R_{i \bar{\jmath}}\right)-\lambda \operatorname{tr} R_{i \bar{\jmath}} .
$$

On the other hand,

$$
g^{\bar{\beta} \alpha}\left(\varphi_{\alpha ; i} \varphi_{\bar{\beta} ; \bar{j}}^{*}\right)=g^{\bar{\beta} \alpha}\left(\varphi_{\alpha} \varphi_{\bar{\beta}}^{*}\right)_{i \bar{\jmath}}-\left(\varphi_{\alpha ; i \bar{\jmath}} \cdot \varphi_{\bar{\beta}}^{*}\right),
$$

and

$$
\varphi_{\alpha ; i \bar{\jmath}}=-\operatorname{tr}\left[R_{i \bar{\jmath}}, \varphi_{\alpha}\right],
$$

from which the claim follows.

From now on, we assume that $X$ is a Kähler manifold whose Kähler form is the Chern form

$$
\omega_{X}=c_{1}\left(\mathcal{L}, h_{\mathcal{L}}\right)
$$

of a positive Hermitian line bundle $\left(\mathcal{L}, h_{\mathcal{L}}\right)$. Note that this implies that $X$ is a complex projective manifold. 
Given a proper, smooth holomorphic map $f: \mathcal{X} \rightarrow S$ and a locally free sheaf $\mathcal{F}$ on $\mathcal{X}$, the determinant line bundle of $\mathcal{F}$ on $S$ is by definition $\operatorname{det} \underline{R} f_{*} \mathcal{F}$.

The generalized Riemann-Roch theorem by Bismut et al. [9] applies to Hermitian vector bundles $(\mathcal{F}, h)$ on $\mathcal{X}$. It states that the determinant line bundle of $\mathcal{F}$ on $S$ carries a Quillen metric, whose Chern form equals the fiber integral

$$
\int_{\mathcal{X} / S} \operatorname{ch}(\mathcal{F}, h) \operatorname{td}\left(\frac{\mathcal{X}}{S}, \omega_{\mathcal{X}}\right) s
$$

where ch and td denote, respectively, the Chern character form and the Todd form (for smooth, proper holomorphic maps over singular base spaces, cf. [10, Appendix]).

We first mention

$$
\operatorname{ch}(\operatorname{End}(\mathcal{E}))=r^{2}+2 r \operatorname{ch}_{2}(\mathcal{E})-c_{1}^{2}(\mathcal{E})+\cdots
$$

so that for the virtual bundle $\operatorname{End}(\mathcal{E})-\mathcal{O}^{r^{2}}$

$$
\operatorname{ch}\left(\operatorname{End}(\mathcal{E})-\mathcal{O}^{r^{2}}\right)=2 r \operatorname{ch}_{2}(\mathcal{E})-c_{1}^{2}(\mathcal{E})+\cdots
$$

holds. We use these formulas for Hermitian bundles now.

$$
\begin{aligned}
\operatorname{ch} & \left.\left((\operatorname{End}(\mathcal{E}), h)-\mathcal{O}^{r^{2}}\right) \otimes\left(\left(\mathcal{L}, h_{\mathcal{L}}\right)-\left(\mathcal{L}^{-1}, h_{\mathcal{L}}^{-1}\right)\right)^{\otimes(n-1)}\right) \\
& =\operatorname{ch}_{2}\left((\operatorname{End}(\mathcal{E}), h)-\mathcal{O}^{r^{2}}\right) \cdot 2^{n-1} \omega_{X}^{n-1}+\cdots \\
& =\left(2 r\left(\frac{1}{2} \operatorname{tr}\left(\frac{\sqrt{-1}}{2 \pi} \Omega \wedge \frac{\sqrt{-1}}{2 \pi} \Omega\right)\right)-\left(\operatorname{tr} \frac{\sqrt{-1}}{2 \pi} \Omega\right)^{2}\right) 2^{n-1} \omega_{X}^{n-1}+\cdots \\
& =2^{n-1}\left(r \operatorname{tr}\left(\frac{\sqrt{-1}}{2 \pi} \Omega \wedge \frac{\sqrt{-1}}{2 \pi} \Omega\right)-\left(\operatorname{tr} \frac{\sqrt{-1}}{2 \pi} \Omega\right)^{2}\right) \omega_{X}^{n-1}+\cdots
\end{aligned}
$$

The highest exterior power $\Lambda^{r} \mathcal{E}$ carries the induced Hermitian metric $\widehat{h}$, for which the following identity holds:

$$
\begin{aligned}
\operatorname{ch} & \left.\left(\left(\Lambda^{r} \mathcal{E}, \widehat{h}\right)-\left(\Lambda^{r} \mathcal{E}, \widehat{h}\right)^{-1}\right)^{\otimes 2} \cdot\left(\left(\mathcal{L}, h_{\mathcal{L}}\right)-\left(\mathcal{L}^{-1}, h^{-1}\right)\right)^{\otimes(n-1)}\right) \\
& =2^{n+1} c_{1}^{2}(\mathcal{E}, h) \cdot c_{1}\left(\mathcal{L}, h_{\mathcal{L}}\right)^{n-1}+\cdots \\
& =2^{n+1} c_{1}^{2}(\mathcal{E}, h) \cdot \omega_{X}^{n-1}+\cdots \\
& =2^{n+1}\left(\operatorname{tr} \frac{\sqrt{-1}}{2 \pi} \Omega\right)^{2} \omega_{X}^{n-1}+\cdots
\end{aligned}
$$

Hence, we have the following theorem: 
Theorem 6.2. The generalized Petersson-Weil form can be expressed in terms of Chern character forms of Hermitian bundles:

$$
\begin{aligned}
\frac{1}{4 \pi^{2}} \omega_{\mathrm{PW}}= & -\frac{1}{2^{n} r(n-1) !} \int \operatorname{ch}\left(\left(\operatorname{End}(\mathcal{E})-\mathcal{O}^{r^{2}}\right) \otimes\left(\mathcal{L}-\mathcal{L}^{-1}\right)^{\otimes(n-1)}\right) \\
& -\frac{1}{2^{n+2} r(n-1) !} \int \operatorname{ch}\left(\left(\Lambda^{r} \mathcal{E}-\left(\Lambda^{r} \mathcal{E}\right)^{-1}\right)^{\otimes 2} \otimes\left(\mathcal{L}-\mathcal{L}^{-1}\right)^{\otimes(n-1)}\right) \\
& +\frac{\lambda}{2 \pi} \frac{1}{2^{n+1} n !} \int \operatorname{ch}\left(\left(\Lambda^{r} \mathcal{E}-\left(\Lambda^{r} \mathcal{E}\right)^{-1}\right) \otimes\left(\mathcal{L}-\mathcal{L}^{-1}\right)^{\otimes n}\right) \\
& +\frac{1}{8 \pi^{2}} \sqrt{-1} \partial \bar{\partial} \int \operatorname{tr}\left(\varphi \wedge \varphi^{*}\right) \wedge \frac{\omega_{X}^{n-1}}{(n-1) !}
\end{aligned}
$$

Let $q: X \times \mathcal{M}_{\mathrm{H}} \rightarrow \mathcal{M}_{\mathrm{H}}$ be the canonical projection. We introduce the following determinant line bundles $\delta_{j}$, equipped with Quillen metrics $h_{j}^{Q}$.

$$
\begin{aligned}
& \delta_{1}=\operatorname{det} \underline{\underline{R}} q_{*}\left(\left(\operatorname{End}(\mathcal{E})-\mathcal{O}^{r^{2}}\right) \otimes\left(\mathcal{L}-\mathcal{L}^{-1}\right)^{\otimes(n-1)}\right) \\
& \delta_{2}=\operatorname{det} \underline{\underline{R}} q_{*}\left(\left(\Lambda^{r} \mathcal{E}-\left(\Lambda^{r} \mathcal{E}\right)^{-1}\right)^{\otimes 2} \otimes\left(\mathcal{L}-\mathcal{L}^{-1}\right)^{\otimes(n-1)}\right) \\
& \delta_{3}=\operatorname{det} \underline{\underline{R}} q_{*}\left(\left(\Lambda^{r} \mathcal{E}-\left(\Lambda^{r} \mathcal{E}\right)^{-1}\right) \otimes\left(\mathcal{L}-\mathcal{L}^{-1}\right)^{\otimes n}\right) .
\end{aligned}
$$

Setting

$$
\chi=\int \operatorname{tr}\left(\varphi \wedge \varphi^{*}\right) \wedge \frac{\omega_{X}^{n}}{(n-1) !}
$$

we equip the trivial bundle $\mathcal{O}_{\mathcal{M}_{\mathrm{H}}}$ with the Hermitian metric $e^{\chi}$.

Theorem 6.3. The generalized Petersson-Weil Kähler form is a linear combination of the $(1,1)$-forms $c_{1}\left(\delta_{j}, h_{j}^{Q}\right), j=1,2,3$, and $c_{1}\left(\mathcal{O}_{\mathcal{M}_{\mathrm{H}}}, e^{\chi}\right)$.

\section{A holomorphic closed 2-form on a moduli space of Higgs bundles}

Let $(\mathcal{E}, \varphi)$ be a universal family of stable Higgs bundles on a Kähler manifold $\left(X, \omega_{X}\right)$ over a complex analytic space $S$ carrying the unique family of Hermitian-Yang-Mills connections $\left\{\theta_{s}\right\}_{s \in S}$. Using the previous notation, we introduce a holomorphic 2 -form $\pi$ on $S$ with $d \pi=0$.

Let $\left.\frac{\partial}{\partial s^{i}}\right|_{s=s_{0}}$ be a tangent vector, and

$$
\begin{aligned}
\rho\left(\left.\frac{\partial}{\partial s^{i}}\right|_{s=s_{0}}\right)= & \varphi_{\alpha ; i} d z^{\alpha}+R_{i \bar{\beta}} d z^{\bar{\beta}} \\
& \in C^{1,0}\left(X \times S, \operatorname{End}\left(\mathcal{E}_{s}\right)\right) \oplus C^{0,1}\left(X \times S, \operatorname{End}\left(\mathcal{E}_{s}\right)\right) .
\end{aligned}
$$


Definition 7.1. A 2-form

$$
\pi=\pi_{i k}(s) d s^{i} \wedge d s^{k}
$$

on $S$ is given by

$$
\pi_{i k}=\int_{X \times\{s\}} \operatorname{tr}\left(g^{\bar{\beta} \alpha}\left(\varphi_{\alpha ; i} \cdot R_{k \bar{\beta}}-\varphi_{\alpha ; k} \cdot R_{i \bar{\beta}}\right)\right) g d V .
$$

Lemma 7.2. The 2-form $\pi$ is holomorphic, and furthermore, it is of the form $\pi=d \nu$ for a certain holomorphic 1-form $\nu$ on $S$. The forms $\nu$ and $\pi$ descend to the moduli space of Higgs bundles as holomorphic forms.

Proof. We define $\nu=\nu_{i} d s^{i}$ through

$$
\nu_{i}=2 \int_{X \times\{s\}} \operatorname{tr} g^{\bar{\beta} \alpha} \varphi_{\alpha} R_{i \bar{\beta}} g d V .
$$

Then, $d \nu=\pi$ follows immediately, and

$$
\frac{\partial \nu_{i}}{\partial s^{\bar{\ell}}}=\int \operatorname{tr} g^{\bar{\beta} \alpha} \varphi_{\alpha} R_{i \bar{\beta} ; \bar{\ell}} g d V=-\int \operatorname{tr} g^{\bar{\beta} \alpha} \varphi_{\alpha ; \bar{\beta}} R_{i \bar{\ell}} g d V=0
$$

completing the proof of the lemma.

Denote by $\mathcal{M}$ the moduli space of stable vector bundles on $X$. As any stable vector bundle $E$ defines a Higgs bundle $(E, \varphi)$ with Higgs field $\varphi=0$. The Hermite-Einstein connection on the stable vector bundle $E$ coincides with the Hermitian-Yang-Mills connection on $(E, 0)$. We have an embedding $i: \mathcal{M} \hookrightarrow \mathcal{M}_{\mathrm{H}}$ into the corresponding moduli space of stable Higgs bundles. Let $\mathcal{M}_{\mathrm{H}}^{s} \subset \mathcal{M}_{\mathrm{H}}$ denote the Zariski open subset defined by all Higgs bundles $(E, \varphi)$ with $E$ stable. Therefore, we have a retraction $f: \mathcal{M}_{\mathrm{H}}^{s} \rightarrow \mathcal{M}$ that sends any $(E, \varphi)$ to $E$.

Proposition 7.3. The forms $\nu$ and $\pi$ vanish on the fibers of $f$.

Proof. On the level of base spaces of universal deformations, $f$ is a submersion of the form $\operatorname{pr}_{1}: S=S^{\prime} \times S^{\prime \prime} \rightarrow S^{\prime}$. Let $s=\left(s^{\prime}, s^{\prime \prime}\right) \in S$. Let $v=\frac{\partial}{\partial s^{i}} \in$ $T_{s} S$ be a tangent vector with

$$
\rho(v)=\varphi_{\alpha ; i} d z^{\alpha}+R_{i \bar{\beta}} d z^{\bar{\beta}}
$$

in the sense of (7.1). Then $\operatorname{pr}_{1}(v)$ is represented by $R_{i \bar{\beta}} d z^{\bar{\beta}}$, and if $v \in$ $T_{s} S^{\prime \prime} \hookrightarrow T_{s} S$, the form $R_{i \bar{\beta}} d z^{\bar{\beta}}$ is $\bar{\partial}$-exact. Conversely, let $R_{k \bar{\beta}}=E_{; \bar{\beta}}$ for 
some section $E$ of $\operatorname{End}\left(\mathcal{E} \mid X \times S^{\prime \prime}\right)$. Then,

$$
\nu_{k}(s)=\int \operatorname{tr} g^{\bar{\beta} \alpha} \varphi_{\alpha} E_{; \bar{\beta}} g d V=0
$$

proving the proposition.

If the form $\nu$ is non-zero on $\mathcal{M}_{\mathrm{H}}^{s}$, then it does not descend under $f$.

\section{Non-Abelian Hodge symmetry, symplectic and hyper-Kähler structure}

Let $(E, \varphi)$ be a stable Higgs bundle over $X$, equipped with a HermitianYang-Mills connection. We first provide the space of infinitesimal deformations of $(E, \varphi)$, namely $\mathbb{H}^{1}\left(C^{\bullet \bullet}\right)$, with a quaternionic structure under an assumption on $\mathbb{H}^{2}\left(C^{\bullet \bullet}\right)$.

Assumption 8.1. For the rest of this section, we restrict ourselves to stable Higgs bundles $(E, \varphi)$ satisfying the following two conditions:

A: The rational characteristic classes of the projective bundle $\mathbb{P}(E)$ over $X$ are assumed to be zero. This is equivalent to the condition that the Hermitian-Yang-Mills connection on $(E, \varphi)$ is projectively flat, i.e.,

$$
R_{\alpha \bar{\beta}}+\left[\varphi_{\alpha}, \varphi_{\bar{\beta}}^{*}\right]=\lambda \cdot g_{\alpha \bar{\beta}} \cdot \operatorname{id}_{E}
$$

for some $\lambda \in \mathbb{C}$.

B: $\operatorname{dim} \mathbb{H}^{2}\left(C^{\bullet \bullet}\right)=1$.

In view of Lemma 3.6, this is equivalent to the condition that

$$
\mathbb{H}^{2}\left(C^{\bullet \bullet}\right)=\mathbb{C} \cdot \omega_{X} \cdot \operatorname{id}_{E}
$$

The above condition A can be replaced by the following condition:

$\mathrm{A}^{\prime}$ : The Higgs field $\varphi$ is $\partial_{\theta}$-closed with respect to the Hermitian-YangMills connection, i.e.,

$$
\varphi_{\alpha ; \gamma}=\varphi_{\gamma ; \alpha}
$$


Involution 8.2. We have an involution of the space of $\operatorname{End}(E)$-valued 1-forms defined by

$$
\begin{aligned}
& \iota: C^{1} \longrightarrow C^{1} \\
& (a, b) \longmapsto\left(-b^{*}, a^{*}\right),
\end{aligned}
$$

where $C^{1}$ is defined in (3.1). Obviously $\iota^{2}=-\mathrm{id}_{C^{1}}$. We shall see this involution descends to the space of infinitesimal deformations of $(E, \varphi)$.

Proposition 8.3. Let $\eta \in C^{1}$ be harmonic (so $\eta$ gives an infinitesimal deformation of $(E, \varphi))$. Then $\iota(\eta)$ is harmonic.

Proof. Let $\eta \neq 0$. Since deformations are not obstructed, we can assume that there is a coordinate system on the base $S$ of a universal deformation so that $\eta$ is of the form $\eta_{i}$ in the sense of (3.8), i.e.,

$$
\iota \eta_{i}=\left(-R_{\alpha \bar{\imath}} d z^{\alpha}, \varphi_{\bar{\beta} ; \bar{\imath}}^{*} d z^{\bar{\beta}}\right) .
$$

Claim 1.

$$
d\left(\iota \eta_{i}\right)=\xi_{; \bar{i}}
$$

where

$$
\xi=\left(\varphi_{\alpha ; \gamma} d z^{\alpha} \wedge d z^{\gamma},\left(R_{\alpha \bar{\beta}}+\left[\varphi_{\alpha}, \varphi_{\bar{\beta}}^{*}\right]\right) d z^{\alpha} \wedge d z^{\bar{\beta}},-\varphi_{\bar{\beta} ; \bar{\delta}}^{*} d z^{\bar{\beta}} \wedge d z^{\bar{\delta}}\right) .
$$

Proof of Claim 1. Let $d\left(\iota \eta_{i}\right)=\left(d\left(\iota \eta_{i}\right)_{1}, d\left(\iota \eta_{i}\right)_{2}, d\left(\iota \eta_{i}\right)_{3}\right)$. Then,

$$
d\left(\iota \eta_{i}\right)_{1}=\left[R_{\alpha \bar{\imath}} d z^{\alpha}, \varphi_{\gamma} d z^{\gamma}\right]=\varphi_{\alpha ; \gamma \bar{\imath}} d z^{\alpha} \wedge d z^{\gamma} .
$$

Next,

$$
\begin{aligned}
d\left(\iota \eta_{i}\right)_{2} & =\bar{\partial}\left(-R_{\alpha \bar{\imath}} d z^{\alpha}\right)+\left[\varphi_{\bar{\beta} ; \bar{\imath}}^{*} d z^{\bar{\beta}}, \varphi_{\alpha} d z^{\alpha}\right] \\
& =\left(R_{\alpha \bar{\beta}}+\left[\varphi_{\alpha}, \varphi_{\bar{\beta} ; \bar{\imath}}^{*}\right)_{; \bar{\imath}} d z^{\alpha} \wedge d z^{\bar{\beta}}\right.
\end{aligned}
$$

and

$$
d\left(\iota \eta_{i}\right)_{3}=\bar{\partial}\left(\varphi \frac{*}{\beta} ; \bar{\imath} d z^{\bar{\beta}}\right)=-\varphi_{\bar{\beta} ; \bar{\delta} \bar{\imath}} d z^{\bar{\beta}} \wedge d z^{\bar{\delta}}
$$

proving Claim 1. 
Claim 2.

$$
d^{*}\left(\iota \eta_{i}\right)=0
$$

Proof of Claim 2.

$$
\begin{aligned}
d^{*}\left(\iota \eta_{i}\right) & =+\Lambda\left[R_{\alpha \bar{\imath}} d z^{\alpha}, \varphi_{\bar{\beta}}^{*} d z^{\bar{\beta}}\right]+\bar{\partial}^{*}\left(\varphi_{\bar{\beta} ; \bar{\imath}}^{*} d z^{\bar{\beta}}\right) \\
& =g^{\bar{\beta} \alpha}\left(\left[R_{\alpha \bar{\imath}}, \varphi_{\bar{\beta}}^{*}\right]-\varphi \varphi_{\bar{\beta} ; \bar{\imath} \alpha}^{*}\right)=0 .
\end{aligned}
$$

Claim 3.

$$
d \xi=0
$$

Proof of Claim 3. Let

$$
d \xi=\left(d \xi_{1}, \ldots, d \xi_{4}\right)
$$

where $d \xi_{i}$ is the $i$-th component of $d \xi$ in the decomposition in (3.1). Then,

$$
d \xi_{1}=\left[-\varphi_{\alpha ; \gamma} d z^{\alpha} \wedge d z^{\gamma}, \varphi_{\sigma} d z^{\sigma}\right]=0
$$

because of (2.1).

Next,

$$
\begin{aligned}
d \xi_{2} & =\bar{\partial}\left(\varphi_{\alpha ; \gamma} d z^{\alpha} \wedge d z^{\gamma}\right)+\left[\left(R_{\alpha \bar{\beta}}+\left[\varphi_{\alpha}, \varphi_{\bar{\beta}}^{*}\right]\right) d z^{\alpha} \wedge d z^{\bar{\beta}}, \varphi_{\gamma} d z^{\gamma}\right] \\
& =\left(\varphi_{\alpha ; \gamma \bar{\beta}}-\left[R_{\alpha \bar{\beta}}, \varphi_{\gamma}\right]-\left[\left[\varphi_{\alpha}, \varphi_{\bar{\beta}}^{*}\right], \varphi_{\gamma}\right]\right) d z^{\alpha} \wedge d z^{\gamma} \wedge d z^{\bar{\beta}} \\
& =\left(\left[\varphi_{\alpha}, R_{\gamma \bar{\beta}}\right]+\left[\varphi_{\gamma}, R_{\alpha \bar{\beta}}\right]-\left[\left[\varphi_{\alpha}, \varphi_{\bar{\beta}}^{*}\right], \varphi_{\gamma}\right]\right) d z^{\alpha} \wedge d z^{\gamma} \wedge d z^{\bar{\beta}}
\end{aligned}
$$

The first two terms together are symmetric in $\alpha$ and $\gamma$, also the third term because of (2.1), so that $d \xi_{2}$ vanishes.

Finally,

$$
d \xi_{3}=-\left[-\varphi_{\bar{\beta} ; \bar{\delta}}^{*}, \varphi_{\alpha}\right] d z^{\bar{\beta}} \wedge d z^{\bar{\delta}} \wedge d z^{\alpha}+\bar{\partial}\left(R_{\alpha \bar{\beta}}+\left[\varphi_{\alpha}, \varphi_{\bar{\beta}}^{*}\right]\right) d z^{\alpha} \wedge d z^{\bar{\beta}}=0
$$

and

$$
d \xi_{4}=\bar{\partial}\left(-\varphi_{\bar{\beta} ; \delta}^{*} d z^{\bar{\beta}} \wedge d z^{\bar{\delta}}\right)=0
$$

proving Claim 3. 
Claim 4.

$$
d^{*} \xi=0
$$

Proof of Claim 4. Let $\xi=(u, v, w)$. Here projective flatness will be used. According to (3.7), the first component of $d^{*} \xi$ equals

$$
d^{*} \xi_{1}=\Lambda\left(\left[\varphi_{\alpha ; \gamma} d z^{\alpha} \wedge d z^{\gamma}, \varphi_{\bar{\delta}}^{*} d z^{\bar{\delta}}\right]\right)+\bar{\partial}^{*} v
$$

where $d^{*} \xi_{i}$ is the $i$-th component of $d^{*} \xi$ in the decomposition in (3.1). Because of (8.1), we have $\bar{\partial}^{*} v=0$, and

$$
d^{*} \xi_{1}=\frac{1}{2} g^{\bar{\beta} \gamma}\left[\left(\varphi_{\alpha ; \gamma}-\varphi_{\gamma ; \alpha}\right), \varphi_{\bar{\beta}}^{*}\right] d z^{\alpha}=\frac{1}{2} g^{\bar{\beta} \gamma}\left(R_{\alpha \bar{\beta} ; \gamma}-R_{\gamma \bar{\beta} ; \alpha}\right) d z^{\alpha}=0
$$

We compute $d^{*} \xi_{2}$. By (8.1), the term $\widetilde{\Lambda}\left[v, \varphi^{*}\right]$ vanishes, and

$$
\begin{aligned}
\bar{\partial}^{*}\left(-\varphi_{\bar{\beta} ; \bar{\delta}}^{*} d z^{\bar{\beta}} \wedge d z^{\bar{\delta}}\right) & =\frac{1}{2} g^{\bar{\delta} \gamma}\left(\varphi_{\bar{\beta} ; \bar{\delta} \gamma}^{*}-\varphi_{\bar{\delta} ; \bar{\beta} \gamma}^{*}\right) d z^{\bar{\beta}} \\
& =\frac{1}{2} g^{\bar{\delta} \gamma}\left(-\left[\varphi_{\frac{\beta}{\beta}}^{*}, R_{\gamma \bar{\delta}}\right]+\left[\varphi_{\frac{\delta}{\delta}}^{*}, R_{\gamma \bar{\beta}}\right]\right) d z^{\bar{\beta}} \\
& =\frac{1}{2} g^{\bar{\delta} \gamma}\left(\left[\left[\varphi_{\bar{\beta}}^{*}, \varphi_{\gamma}\right], \varphi_{\bar{\delta}}^{*}\right]-\left[\left[\varphi_{\bar{\delta}}^{*}, \varphi_{\gamma}\right], \varphi_{\bar{\beta}}^{*}\right]\right) d z^{\bar{\beta}}
\end{aligned}
$$

This form vanishes because of the integrability condition (2.1), proving Claim 4. The computation is the same with $\mathrm{A}$ replaced by $\mathrm{A}^{\prime}$.

Now, by assumption $\mathrm{B}$, the harmonic form $\xi$ is of the form $c(s) \cdot \omega_{X} \operatorname{id}_{E}$. From the definition of $\xi$ and (8.1), we know that $c(s)=\lambda$ is independent of $s \in S$. Hence, $d\left(\iota \eta_{i}\right)=0$. This completes the proof of the proposition.

Corollary 8.4. The d-closed holomorphic two-form $\pi$ is non-degenerate at $(E, \varphi)$. In particular, the dimension of $\mathbb{H}^{1}\left(C^{\bullet \bullet}\right)$ is even.

Proof. In the above notation,

$$
\pi_{i k}=\left\langle\eta_{k}, \iota\left(\eta_{i}\right)\right\rangle
$$

As $\iota$ takes harmonic elements of $C^{1}$ to harmonic elements of $C^{1}$, it induces a bijective map of $\mathbb{H}^{1}$ to itself. This shows the non-degeneracy.

Remark 8.5. For $X=\mathbb{C P}^{2}$, there are examples of stable Higgs bundles where $\operatorname{dim} \mathbb{H}^{1}\left(C^{\bullet \bullet}\right)$ is odd. (They do not satisfy the two assumptions.) 
We insert an observation about quaternions: Assume that $(\mathbb{H}, I, J, K)$ is identified with $\mathbb{C}^{2}=\{(z, w)\}$ in such a way that $I(z, w)=(i z, i w), i=$ $\sqrt{-1}$. Namely, we identify $(z, w)=(x+i y, u+i v)$ with $x+I y+(u+I v) J$. Then, $J(z, w)=(-\bar{w}, \bar{z})$, and $K(z, w)=I J(z, w)=(-i \bar{w}, i \bar{z})$.

This suggests the following almost quaternionic structure on $\mathbb{H}^{1}\left(C^{\bullet \bullet}\right)$. For $\eta=(a, b) \in \mathbb{H}^{1}\left(C^{\bullet \bullet}\right)$, we have $\iota(a, b)=\left(-b^{*}, a^{*}\right)$, and hence for a tangent vector

$$
I(\eta)=\sqrt{-1} \cdot \eta, \quad J(\eta)=\iota(\eta), \quad K(\eta)=\sqrt{-1} \cdot \iota(\eta) .
$$

By definition, the equations for an almost quaternionic structure are verified.

For complex tangent vectors $\eta, \vartheta$ of the base space have

$$
\begin{aligned}
\langle I \eta, \vartheta\rangle & =\omega^{\mathrm{PW}}(\eta, \vartheta), \\
\langle J \eta, \vartheta\rangle & =\bar{\pi}(\eta, \vartheta), \\
\langle K \eta, \vartheta\rangle & =i \bar{\pi}(\eta, \vartheta),
\end{aligned}
$$

where $\bar{\pi}$ denotes the conjugate of $\pi$. The corresponding differential forms are closed (and non-degenerate), so that together with [1, Lemma (6.8)] the following holds:

Theorem 8.6. Consider the Zariski open subset $W$ of the moduli of stable Higgs bundles over which the condition $\mathbb{H}^{2}=\mathbb{C} \cdot \omega_{X} \mathrm{id}_{E}$ holds. Assume that either the Hermitian-Yang-Mills connections are projectively flat or that the Higgs fields are $\partial_{\theta}$-closed with respect to the Hermitian-Yang-Mills connection. Then $W$ carries a natural hyper-Kähler structure, related to the Petersson-Weil structure $\omega^{\mathrm{PW}}$ and the holomorphic symplectic form $\pi$.

We note that our proof shows more. It gives the following proposition.

Proposition 8.7. Consider the moduli space of Higgs bundles of the form $(E, \varphi)$, where the determinant bundle of $E$ is a fixed line bundle and where $\operatorname{trace}(\varphi)=0$. This moduli space carries a natural hyper-Kähler structure, related to the Petersson-Weil structure $\omega^{\mathrm{PW}}$ and the holomorphic symplectic form $\pi$, provided condition $\mathrm{A}$ or condition $\mathrm{A}^{\prime}$ holds along with the following weaker form of condition $\mathrm{B}$ :

$\mathrm{B}^{\prime}$ :

$$
\mathbb{H}^{2}\left(D_{0}^{\bullet}\right)=0,
$$

where $D_{0}^{\bullet}$ is the complex in Lemma 3.5 . 


\section{Acknowledgments}

The first named author wishes to thank the University of Marburg for its hospitality and support, while the second named author would like to thank the Tata Institute for Fundamental Research for hospitality and support. This work was partially supported by DFG-Schwerpunktprogramm "Globale Methoden in der Komplexen Geometrie".

\section{References}

[1] N. Hitchin, The self-duality equations on a Riemann surface, Proc. Lond. Math. Soc. 55 (1987), 59-126.

[2] C. T. Simpson, Constructing variations of Hodge structure using YangMills theory and applications to uniformization, J. Amer. Math. Soc., 1 (1988), 867-918.

[3] C. T. Simpson, Moduli of representations of the fundamental group of a smooth projective variety. II, Publ. Math. I.H.É.S., 80 (1994), 5-79.

[4] G. Schumacher and M. Toma, On the Petersson-Weil metric for the moduli space of Hermite-Einstein bundles and its curvature, Math. Ann., 293 (1992), 101-107.

[5] I. Biswas and G. Schumacher, Determinant bundle, Quillen metric, and Petersson-Weil form on moduli spaces, Geom. Funct. Anal., 9 (1999), 226-256.

[6] G. Schumacher, Moduli as algebraic spaces. Complex analysis in several variables-Memorial Conference of Kiyoshi Oka's Centennial Birthday, Adv. Stud. Pure Math., 42, Math. Soc. Japan, Tokyo, 2004, 283-288.

[7] I. Biswas, A remark on a deformation theory of Green and Lazarsfeld, J. Reine Angew. Math., 449 (1994), 103-124.

[8] I. Biswas and S. Ramanan, An infinitesimal study of the moduli of Hitchin pairs, J. Lond. Math. Soc., 49 (1994), 219-231.

[9] J.-M. Bismut, H. Gillet, and C. Soulé, Analytic torsion and holomorphic determinant bundles. I: Bott-Chern forms and analytic torsion. II: Direct images and Bott-Chern forms. III: Quillen metrics on holomorphic determinants, Commun. Math. Phys., 115 (1988), 49-78, 79-126, 301-351. 
[10] A. Fujiki and G. Schumacher, The moduli space of compact extremal Kähler manifolds and generalized Petersson-Weil metrics, Publ. RIMS, Kyoto Univ., 26 (1990), 101-183.

[11] C. T. Simpson, Higgs bundles and local systems, Publ. Math. I.H.É.S., 75 (1992), 5-95.

[12] J. Varouchas, Stabilité de la classe des variétés Kählériennes par certains morphismes propres, Invent. Math., 77 (1984), 117-127.

School of Mathematics

Tata Institute of Fundamental Research

HOMI BHABHA ROAD

BOMBAY 400005

INDIA

E-mail address: indranil@math.tifr.res.in

FACHBEREICH MATHEMATIK UND INFORMatiK

Philipps-Universität MARBurG

LAHNBERGE

Hans-MeERWein-Strasse

D-35032 MARBURG

Germany

E-mail address: schumac@mathematik.uni-marburg.de

Received September 21, 2005 
